\title{
Expression of IL-5 alters bone metabolism and induces ossification of the spleen in transgenic mice
}

\author{
MiMi P. Macias, ${ }^{1}$ Lorraine A. Fitzpatrick, ${ }^{2}$ Ina Brenneise,${ }^{1}$ Michael P. McGarry, ${ }^{1}$ \\ James J. Lee, ${ }^{1}$ and Nancy A. Lee ${ }^{1}$ \\ ${ }^{1}$ Department of Biochemistry and Molecular Biology, Mayo Clinic Scottsdale, Scottsdale, Arizona, USA \\ ${ }^{2}$ Departments of Medicine and Endocrinology and Metabolism, Mayo Clinic Rochester, Rochester, Minnesota, USA
}

Address correspondence to: Nancy A. Lee, Mayo Clinic Scottsdale, Department of Biochemistry and

Molecular Biology, 13400 E. Shea Boulevard, Scottsdale, Arizona 85259, USA.

Phone: (480) 301-7183; Fax: (480) 301-7017; E-mail: nlee@mayo.edu.

Received for publication September 1, 2000, and accepted in revised form February 5, 2001.

\begin{abstract}
We have developed a transgenic mouse line, NJ.1638, which expresses high levels of IL-5 from T cells, with profound hematological consequences. Eosinophils comprise more than $60 \%$ of circulating white blood cells in these animals, with the total peripheral white blood cell counts increasing more than 40-fold relative to wild-type littermates. This extraordinary proliferative capacity is sustained by expanded sites of extramedullary hematopoiesis and is accompanied by multifocal, ectopic bone formation in the spleen. Histology of the splenic nodules revealed the presence of osteoid matrices and osteocytes trapped within mineralized trabecular plates. In addition, polarized light microscopy of calcified tissue sections revealed both woven bone and areas of organized lamellar bone. Morphometric assessments demonstrated that both the growth and mineralization of splenic bone occurred at rates nearly an order of magnitude higher than in skeletal bone. Skeletal bone metabolic parameters were also perturbed. We also observed heterotopic ossification of the spleen and perturbation of skeletal bone homeostasis following adoptive engraftment of transgenic marrow to wild-type recipients. These data suggest that IL-5 overexpression mediates bone formation through the mobilization of marrowderived osteogenic progenitors and/or the inhibition of recruited osteoclasts.
\end{abstract}

J. Clin. Invest. 107:949-959 (2001).

\section{Introduction}

The close physical association of hematopoietic cells and the bone matrix of the marrow cavity provides a microenvironment supportive of cell-cell and cellmatrix interactions that influence the differentiation of both hematopoietic $(1,2)$ and osteogenic (3) cells. Osteogenic precursor cells are an important component of the bone marrow stroma (4-6), and several studies have demonstrated specific interactions among these cells during bone morphogenesis (7-9). Bone homeostasis is a dynamic process that depends on a balance between bone-forming activities of osteoblasts derived from stromal mesenchymal cells and bone resorption mediated by hematopoietically derived osteoclasts. The differentiation and gene expression patterns of both cell types have been investigated (10). Nonetheless, many of the unique molecular signals regulating the activity of these cells during embryogenesis, skeletal remodeling, or repair are still not well understood. Likely candidate molecules regulating osteoblast/osteoclast activity include a class of secreted signaling molecules known as bone morphogenetic proteins (BMPs). These proteins are members of the TGF- $\beta$ superfamily and have been shown to possess osteoinductive activities $(11,12)$. Additionally, changes in the expression pattern of steroid hormones, cytokines, and growth factors have each been correlated with effects on bone homeostasis, suggesting that multiple osteogenic regulatory pathways exist (13-16).

Bone development occurs by two fundamentally different processes. During embryonic skeletogenesis, intramembranous ossification proceeds in the developing flat bones of the cranial vault, sternum, and pelvis through mesenchymal tissue condensations and differentiation of cells directly to osteoblasts; differentiated osteoblasts synthesize and secrete the bone matrix (i.e., osteoid), which later becomes calcified (13-16). Alternatively, endochondral ossification is the process that occurs in the long bones of the skeleton, whereby mesenchymal cells initially differentiate into cells of the chondrogenic lineage. A transient cartilage scaffold is formed that becomes invaded by hematopoietic components, inducing the recruitment and/or differentiation of mesenchymal cells into osteoblasts. Ultimately, mineralization of osteoid results in replacement of the cartilage matrix with ossified bone (17). The immature bone that forms during both intramembranous and endochondral ossification is woven bone, which is later remodeled to form either compact or cancellous lamellar bone.

Extraskeletal calcification and ossification have been described in association with a variety of disorders. 
Whereas ectopic calcification results in tissue deposition of amorphous calcium phosphate, ectopic ossification results in "true bone" formation, and the mature osseous lesions appear to have both haversian systems and cancellous bone. Clinically, patients have been described with heterotopic ossifications of cutaneous $(18,19)$, intratendinous (20), pulmonary (21), and cochlear and vestibular lumenal (22) sites. Severe examples of dysregulated bone formation include myositis ossificans, a progressive ossification of striated muscle and faciitis (23), and fibrodysplasia ossificans progressiva, which refers to postnatal heterotopic ossification of connective tissue (24). Interestingly, although some of the conditions result from rare genetic disorders (23, $24)$, in others the ectopic bone formation in the skin, muscle, ligaments, and tendons occurs in the context of an initial inflammatory response after injury, surgery, infection, or trauma $(18,20)$. Whether mesenchymal cells with osteogenic potential are endogenous or are recruited to the ossifying tissue in these conditions is an unresolved issue. In addition, although BMP-4 expression has been associated with some of these conditions (e.g., fibrodysplasia ossificans progressiva; ref. 23), the identification of specific growth and/or morphogenic factors that signal postnatal ossification during these disease states remains unclear.

We have generated a transgenic mouse line, NJ.1638, which overproduces the cytokine IL-5 from T cells (25). These mice exhibit profound alterations in hematopoiesis compared with wild-type animals. Given that the spleen remains a source of extramedullary hematopoiesis throughout the lifetime of a mouse, the hematopoietic changes occurring in the transgenic animals have dramatic consequences on this organ. Massive splenomegaly occurs and often results in spleens that weigh in excess of $3 \mathrm{~g}$ (i.e., >10\% of total body weight). In this report, we show that these remarkable changes in the spleen are accompanied by the development of bone nodules, which are often mineralized. Evidence is presented that perturbations in skeletal bone metabolism also occur in these mice. Interestingly, splenic bone formation and skeletal bone perturbations occur in lethally irradiated recipient wild-type animals after engraftment with bone marrow cells from transgenic mice. These observations suggest that a causative link exists between IL-5 expression and bone homeostasis, highlighting a previously unidentified pathway of osteogenesis.

\section{Methods}

Production and preliminary characterization of the NJ.1638 transgenic mouse line. Development of the IL-5 expression construct and production of the NJ.1638 transgenic mouse line have been described previously (25). Briefly, the promoter and tissue-specific enhancer of the murine CD3 $\delta$ gene (26) were used to direct expression of murine IL-5 in peripheral T cells. Transgenic mice were generated by injection of this construct into fertilized eggs derived from crosses of $(\mathrm{C} 57 \mathrm{BL} / 6 \mathrm{~J})$ males to $\mathrm{F}_{1}$
(CBA/J X C57BL/6J) females and have been maintained by continuous backcrossing to $\mathrm{C} 57 \mathrm{BL} / 6 \mathrm{~J}$ mice. The hematopoietic consequences of IL-5 overexpression on the spleens of NJ.1638 mice were assessed from singlecell suspensions prepared by cutting spleens into small pieces, followed by passage through a 22-mesh stainless steel screen. Counts of leukocyte types were determined as the product of the differential percentage derived from Wright-Giemsa-stained cytocentrifuge preparations and the total splenocyte count.

Whole-mount stains of spleens using alizarin red and alcian blue. Spleens from NJ.1638 mice and wild-type littermates were fixed in $100 \%$ ethanol for 4 days and transferred to acetone for 3 days. Adapting a method routinely used for differential staining of bone in intact mouse embryos (27), spleens were gently agitated in staining solution containing $0.001 \%$ alizarin red $\mathrm{S}$ and $0.003 \%$ alcian blue in acidified $(1 \% \mathrm{HCl})$ ethanol for 5 days. Stained spleens were cleared by incubation at room temperature in $20 \%$ glycerol/1\% KOH. Clearing solution was replaced once a week until the transgenic spleen was adequately cleared for photography ( 290 days). Dark-field photomicrographs were taken using a Leica MZ6 dissecting microscope (Leica Microsystems Inc., Deerfield, Illinois, USA).

General histology. Spleens from age- and sex-matched transgenic and wild-type mice were subjected to the following three general histological protocols: (a) Hematoxylin/eosin staining. Before paraffin embedding, transgenic spleens were decalcified in RDO Bone Decalcifier (APEX, Aurora, Colorado, USA) for $45 \mathrm{~min}$ utes at room temperature. Four-micron sections were cut for routine hematoxylin/eosin staining. (b) Goldner's-Masson-Trichrome staining. (c) Toluidine blue staining and polarized light microscopy. These staining procedures were performed on undecalcified tissue sections using routine methodologies $(28,29)$. Undecalcified specimens of either spleen tissue or bone were embedded in modified methylmethacrylate (MMA) (30). Briefly, biopsies were embedded using a temperature-controlled method (Ranier Technical Products, Seattle, Washington, USA) to infiltrate specimens for 4 days in a mixture of $81 \%$ (vol/vol) uninhibited MMA, $8 \%$ (wt/vol) polyethylene glycol distearate, 6.5\% (vol/vol) 2-hydroxy-ethylmethacrylate, 4\% dibutylphthalate, and $0.6 \%$ benzoyl peroxide. Specimens were subsequently placed in fresh monomer-containing accelerator (JB-4; Polysciences, Warrington, Pennsylvania, USA) and polymerized onto aluminum chucks at room temperature in the presence of nitrogen. For polarized light microscopy, stained specimens were observed with a Nikon microphot FXA (Nikon Inc., Melville, New York, USA) using two orthogonally polarized light waves transversing the specimen.

$R T-P C R$. Total RNA was prepared from the spleens of age-matched wild-type and NJ.1638 mice, and from focal sites of apparent bone formation (i.e., splenic nodules [SNs]) individually collected with forceps. Spleen parenchyma samples were minced into 1 - to $3-\mathrm{mm}$ pieces and further dissociated by mechanical shearing 
between the frosted ends of two glass microscope slides and passage through $40 \mu \mathrm{m}$ Nitex screens (TETKO Inc., Kansas City, Missouri, USA). Total RNA from skeletal bones (femur/tibia) and ossified SNs were prepared by freezing the samples in liquid nitrogen and then grinding the frozen tissues with mortar and pestle. Total RNA was recovered from tissue/bone samples using TRI-Reagent (Molecular Research Center Inc., Cincinnati, Ohio, USA) following the manufacturer's protocol. The recovered RNA was treated with DNase I (Life Technologies Inc., Gaithersburg, Maryland, USA) prior to RT using an anchored oligo-dT primer mixture: $\mathrm{dT}_{16} \mathrm{VN}$, where $\mathrm{V}=\mathrm{A}, \mathrm{C}$, or $\mathrm{G}$; and $\mathrm{N}=\mathrm{A}, \mathrm{C}, \mathrm{G}$, or $\mathrm{T}$. PCR was performed in $50 \mu \mathrm{l}$ reaction volumes with cDNA derived from $60 \mathrm{ng}$ of total RNA (35 ng for actin controls). Amplification conditions included $200 \mathrm{nM}$ dNTPs, 5\% DMSO, $0.08 \mu \mathrm{M}$ of each appropriate primer, and 1.5 units of Taq polymerase (Roche Molecular Biochemicals, Basel, Switzerland). Cycling conditions used for PCR: $95^{\circ} \mathrm{C}, 5$ minutes; 30 cycles $\left(95^{\circ} \mathrm{C}, 1\right.$ minute; $50^{\circ} \mathrm{C}$, 1 minute, $72^{\circ} \mathrm{C}, 1$ minute); and $72^{\circ} \mathrm{C}, 7$ minutes. Products were separated through $1 \%$ agarose $/ 0.5 \mathrm{X}$ Tris/borate/EDTA gels and visualized with ethidium bromide. The gene-specific primer pairs were derived from sequences deposited in the GenBank database
(Accession numbers: osteocalcin L24431, actin M12481). Osteocalcin forward primer: 5'-CTAGCAGACACCATGAGGACC-3'; osteocalcin reverse primer: 5'GGTCCTAAATAGTGATACCG-3'; actin forward primer: $5^{\prime}$ GTGGGCCGCTCTAGGCACCA-3'; and actin reverse primer: 5'-TGGCCTTAGGGTTCAGGGGG-3'.

Contact microradiography and measurement of calcium content of the transgenic spleen. To determine the extent of calcification within spleen tissue, sections of MMAembedded specimens were cut to $200 \mu \mathrm{m}$ using an Isomet saw and were placed on emulsion-coated slides (Eastman Kodak Co., Rochester, New York, USA). Sections were exposed to $\mathrm{x}$-ray $(20 \mathrm{kV})$ for 5 minutes and developed according to manufacturer's recommendations. Energy-dispersive $\mathrm{x}$-ray microanalysis was performed to determine chemical composition of the calcified tissue within spleen nodules using undecalcified MMA-embedded spleen sections $(200 \mu \mathrm{m})$ mounted on aluminum stubs with colloidal graphite. Ten spectra were acquired for each sample using a live-count time of 120 seconds. Calcium-to-phosphorus molar ratios were compared to a known standard of hydroxyapatite (Sigma Chemical Co., St. Louis, Missouri, USA).

Histomorphometric measurements of bone growth and mineralization. Age- and sex-matched wild-type and trans-
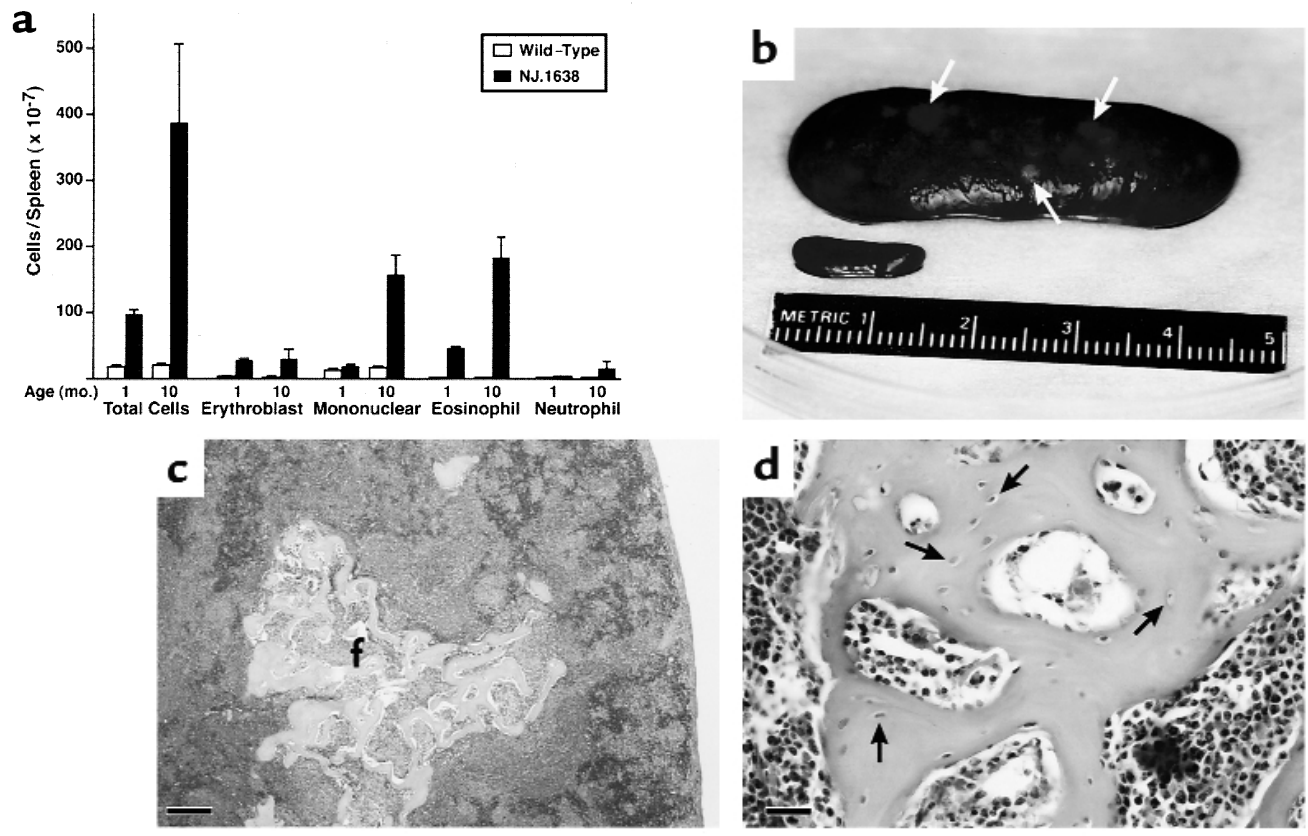

\section{Figure 1}

Constitutive IL-5 expression induces pathological changes in the spleen. (a) Age-dependent cellularity changes in the spleens of wild-type and NJ.1638 mice. Single-cell suspensions were prepared from spleens of wild-type and transgenic mice at 1 month of age (wild-type [ $n=3]$; NJ.1638 $[n=6])$ and 10 months of age (wild-type $[n=3]$; NJ.1638 $[n=4]$ ). Total cell counts $(\geq 200)$ are represented as histograms and are expressed as group mean data $( \pm$ SD). (b) Gross macroscopic comparison of the spleens from age- and sex-matched wild-type and NJ.1638 mice. The wildtype spleen is in the foreground. This comparison demonstrates the extreme splenomegaly and numerous white nodules/foci (arrows) that develop in adult NJ.1638 mice. The wet weight of the transgenic spleen ( $3.75 \mathrm{~g}$ ) is more than 50 times that of the wild-type spleen. (c) Representative hematoxylin/eosin-stained section from a NJ.1638 spleen. This section exhibits a loss of normal spleen histology (i.e., white pulp and red pulp) and reveals the underlying ultrastructure of the white foci ( $f$ ) appearing in $\mathbf{b}$. Scale bar $=160 \mu \mathrm{m}$. (d) Higher magnification of the splenic nodule shown in c demonstrates the unique bone morphology of this structure including the presence of osteocytes (filled arrows) that occupy lacunae of the bone matrix. Scale bar $=20 \mu \mathrm{m}$. 

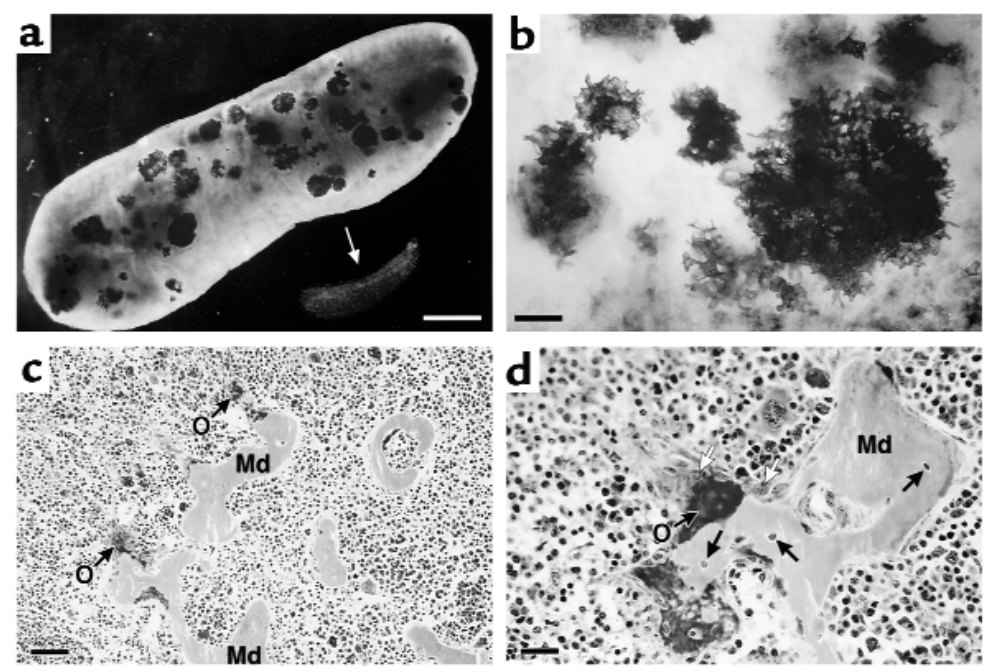

\section{Figure 2}

The multifocal nodules of NJ.1638 spleens exhibit extensive staining for bone but not cartilage. (a) Dark-field photomicrograph of the wildtype and NJ.1638 spleens shown in Figure 1b after whole-mount staining with alizarin red/alcian blue to identify mineralized bone/cartilage, respectively. The wild-type spleen (arrow) displayed no staining with either reagent. In contrast, the numerous, and randomly distributed, nodules of the transgenic spleen stained heavily with alizarin red, providing evidence that these structures are centers of mineralization. Scale bar $=5 \mathrm{~mm}$. (b) Higher magnification shows individual nodules exhibiting fine serpiginous extensions and a distinct lattice-like ultrastructure. Scale bar $=1 \mathrm{~mm}$. (c) NJ.1638 splenic nodules represent areas of active bone formation/mineralization. Photomicrograph of a section from a transgenic spleen following Goldner's staining. These nodules display patterns consistent with bone as characterized by the presence of newly formed unmineralized bone (i.e., red/orange staining osteoid [O]) and green-staining areas of mineralized bone matrix $(\mathrm{Md})$. Scale bar $=50$ $\mu \mathrm{m}$. (d) High-magnification view of Goldner's-stained nodules demonstrates the presence of both bone-forming osteoblasts (open arrows) at the periphery of the nodules, identified as relatively large cells with abundant basophilic cytoplasm and a pale stained nucleus. Osteocytes are seen as embedded cells within the mineralized matrix (filled arrows). Scale bar $=20 \mu \mathrm{m}$.

genic mice, 5-8 months of age, were administered 20 mg of calcein per kilogram of body weight by intramuscular injection. Ten days later, the mice received an intramuscular injection of $20 \mathrm{mg}$ of tetracycline per kilogram of body weight. Two days after the second injection, the spleens and hind limbs were collected and fixed by dehydration through an ascending series of ethanol. Fixed undecalcified tissues were embedded in MMA as already described here, and 5- $\mu \mathrm{m}$ sections were evaluated by fluorescence microscopy (31-33). A standard sampling site was established in the secondary spongiosa of the metaphyseal region of the proximal tibia. This site is $1 \mathrm{~mm}$ distal to the calcein label deposited in the mineralizing cartilage at the epiphyseal growth plate at a point where its center was perpendicular to the long axis of the bone and extends bilaterally in each section (excluding cortical edges). This method compensates for differences in longitudinal growth between control and transgenic mice, to ensure comparable sampling sites and measurement of only remodeling bone. For each section, a total metaphyseal area of $2.8 \mathrm{~mm}^{2}$ was sampled. The mineral apposition rate was calculated using the mean distance in micrometers between the calcein and tetracycline labels (measured every $50 \mu \mathrm{m}$ ) divided by the labeling interval of 10 days, providing a weighted average for the mineral appositional rate. Cancellous bone area was determined as the area of total cancellous bone per square millimeter of metaphyseal area within the sampling site and expressed as a percentage. The bone formation rate (tissue referent) was calculated as the double-labeled perimeter plus one-half single-labeled perimeter per square millimeter of metaphyseal area times the mineral appositional rate. Osteoblasts are designated as a palisade of large basophilic cuboidal cells directly lining the bone perimeter. Student unpaired $t$ tests were used to compare wild-type and transgenic mice. $P<0.05$ was determined to be the level necessary to achieve statistical significance of differences.

Sandwich ELISA assays of cytokine levels. Serum was prepared from peripheral blood ( $0.5 \mathrm{ml}$ per mouse) obtained from the tail. Blood was allowed to form clots at room temperature for 30-60 minutes and was then centrifuged (200 $\mathrm{g}$ for 8 minutes) to obtain cleared supernatants. Serum levels of IL-1 $\beta$, IL-4, IL-5, and IL6 were measured using cytokine-specific ELISA kits (Endogen Inc., Woburn, Massachusetts, USA). Colorometric data was collected with a Molecular Devices $v_{v \max }$ microplate reader (Menlo Park, California, USA) and analyzed using SOFTmax PRO, version 2.2.1 (Molecular Devices Corp., Sunnyvale, California, USA). Statistical analyses were performed using the two-sample $t$ test assuming unequal variances. Cytokine levels are reported as the mean \pm SEM.

Bone marrow transplantation. Adoptive engraftment of hematopoietic cells into $\gamma$-irradiated wild-type mice was accomplished using donor marrow derived from 4month-old male NJ.1638 transgenic mice. The donor marrow was flushed (using PBS) from surgically excised femurs and tibias, aspirated to single cells, and resus- 
pended to $2 \times 10^{7}$ viable cells per milliliter. This marrow suspension was composed of more than $68 \%$ eosinophils, determined on a Diff-Quik-stained cytospin (Shandon Cytospin II; Fisher Scientific Co., Pittsburgh, Pennsylvania, USA) preparation. Recipient wild-type (strain: C57BL/6J) female mice were supralethally irradiated $(1,100 \mathrm{cGy})$ with a sealed ${ }^{137}$ Cesium source (GammaCell Irradiator; MDS Nordion, Kamata, Ontario, Canada). Recipient mice were injected with $10^{7}$ viable cells via a lateral tail vein within 3 hours of irradiation. The percent of male donor engraftment was assessed in recipient female mice using PCR and a Y-chromosome specific primer set (34). Phenotypic assessments of engrafted mice were performed on animals displaying greater than $85 \%$ donor marrow.

\section{Results}

Constitutive expression of IL-5 results in a dramatic expansion of extramedullary hematopoiesis and leads to ectopic bone formation in the spleen

The hematopoietic capacity of an NJ.1638 animal continuously increases during its lifetime such that by 10 months of age total peripheral white blood cell counts increase more than 40 -fold relative to wild-type littermates (>400,000 cells per millimeter cubed; $\sim 60 \%$ eosinophils). This increase is paralleled by concomitant age-dependent increases in both extramedullary hematopoiesis (Figure 1a) and splenomegaly (Figure $1 b)$. In addition to the dramatic increase in the size of transgenic spleens, these organs develop numerous small, hard white foci/nodules visible on the surface (arrows in Figure 1) that are distinct from the splenic parenchymal tissue (Figure 1b).

We previously reported that NJ.1638 spleens undergo histopathological changes that result in the loss of the $\mathrm{red} /$ white pulp architecture and perturbations of the characteristic lymphoid/hematopoietic organization of normal spleen (25). These histological changes are seen in cross-sectional views through decalcified NJ.1638 spleens stained with hematoxylin and eosin (Figure 1c). This spleen section also includes a portion of one of the white splenic foci/nodules indicated in Figure 1b. This serpiginous tissue is delineated from the surrounding cells of the splenic parenchyma and at higher magnification displays morphological characteristics consistent with bone. The decalcified matrix stains strongly with eosin and the cells entrapped within the otherwise acellular matrix of the nodule appear to be osteocytes with densely stained, irregular nuclei (Figure 1d).

The NJ.1638 splenic nodules are histologically indistinguishable from bone

To demonstrate unequivocally that the splenic nodules are bone, several independent assessments of the nodules were performed.

Whole-mount staining for bone/cartilage. The distribution and staining characteristics of the spleen nodules were assessed by a skeletal whole-mount staining technique using alizarin red and alcian blue to distinguish regions of mineralized bone from cartilage, respectively. Wholemount staining showed that the wild-type spleen from an 11-month-old mouse did not contain regions of either cartilage or mineralized bone (Figure 2a, arrow). In contrast, an age-matched transgenic spleen displayed numerous nodules, distributed throughout the tissue, that stained strongly with alizarin red, consistent with mineralized tissue. The nodules were heterogeneous in size, ranging up to $3 \mathrm{~mm}$ in diameter. The absence of alcian blue staining within these mineralized foci, and the transgenic spleen in general, suggests that sulfated glycosaminoglycans (i.e., cartilage) are not a major constituent of the splenic nodules. Higher magnification of the foci revealed the lattice-like structural details of this serpiginous tissue (Figure $2 \mathrm{~b}$ ).

Goldner's staining. The splenic nodules were further characterized as centers of ossification by preparation of undecalcified specimens in MMA and histological staining to identify hallmark features unique to bone, including the presence of osteoblast and osteocyte cell types and the formation of osteoid. The presence of these cell types distinguishes the highly regulated process of bone formation from simple mineralization of the extracellular matrix by calcium deposition. Goldner's-Masson-Trichrome stain, originally developed for microscopic evaluation of undecalcified bone biopsies, clearly showed the presence of osteoid (red/orange areas) and mineralized bone (green areas) within the transgenic spleens (Figure 2c). This staining pattern was unique to transgenic mice, as wild-type spleens failed to display any bone-related characteristics. The stained splenic nodules of transgenic mice were associated with cells that appear to be osteoblasts, and osteocytes were embedded within the calcified matrix (Figure 2d). Additional areas of red/orange staining cells were numerous throughout the parenchyma of transgenic spleens. These areas also appear to be osteoid as systematic assessments at high magnification (i.e., 1,000x) revealed that many, but not all, were associated with detectable traces of greenstained mineralizing zones. No evidence of cartilage was observed in these sections.

Osteoblast-specific gene expression was detected in transgenic spleens. The presence of active osteoblast-like cells in the transgenic spleen was confirmed by using RT-PCR to detect transcripts from the osteocalcin gene, a late osteoblast-specific marker (35). Total RNA from wildtype and transgenic spleen parenchymal tissues, as well as the transgenic SNs, was used to generate cDNA templates for PCR-based assays using an osteocalcin-specific primer pair. Osteocalcin transcripts were not detected in the wild-type spleen or NJ.1638 spleen parenchyma samples, but were uniquely detected in the tissues associated with NJ.1638 SNs (Figure 3a).

Evidence of woven and lamellar bone structure under polarized light. Given that the collagen of bone forms lamellar bands during mineralization and uniquely refracts polarized light, calcified splenic nodules were evaluat- 
a
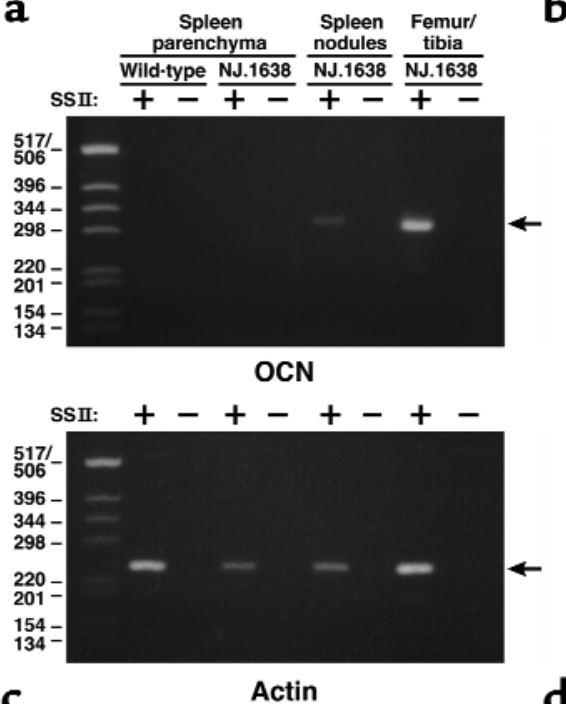

c

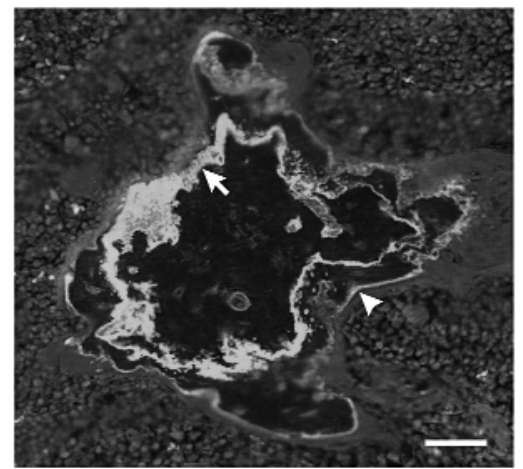

d b
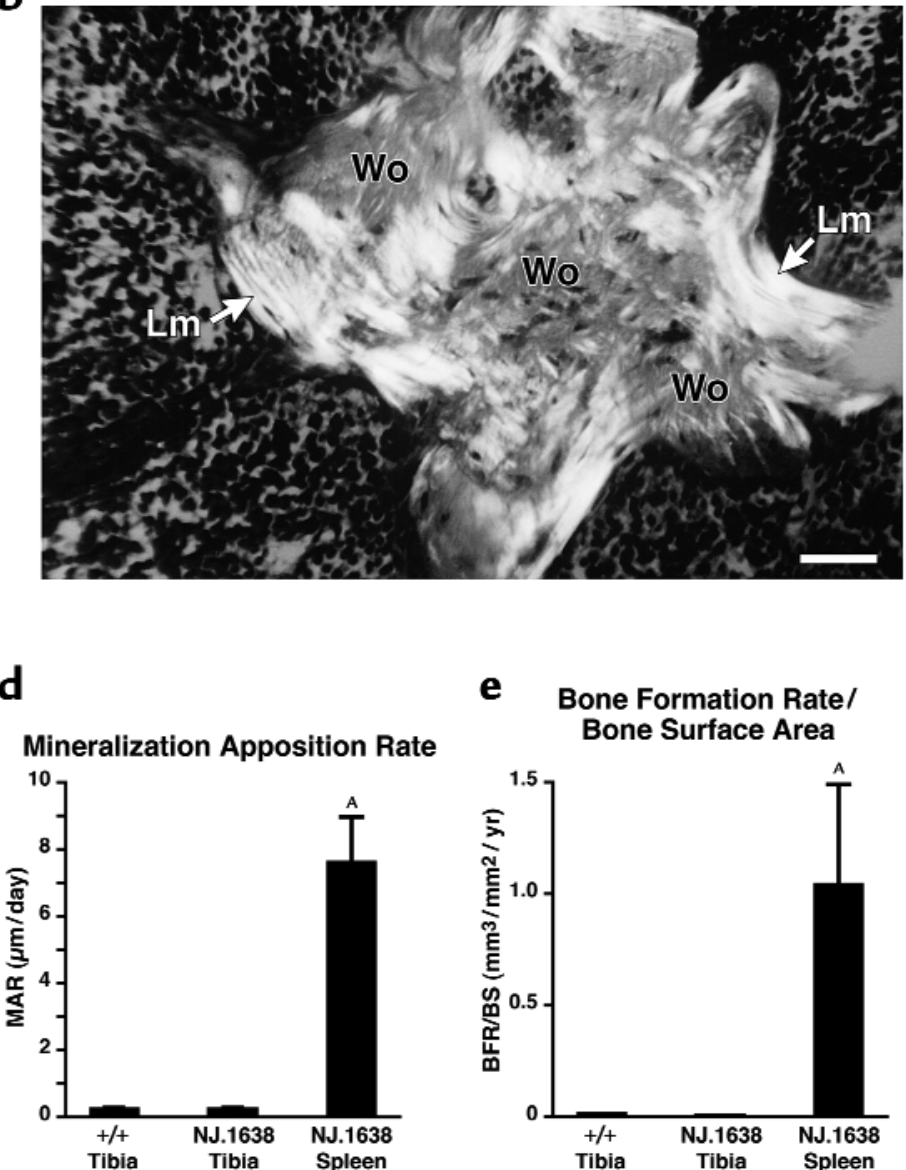

e Bone Formation Rate/ Bone Surface Area

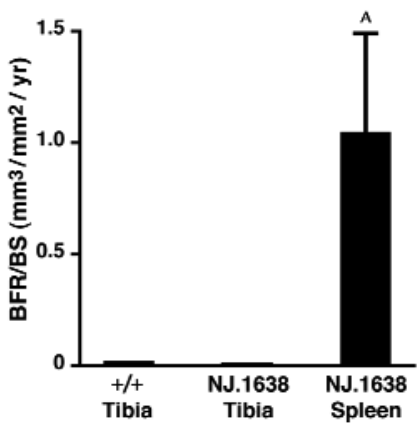

\section{Figure 3}

The SNs of NJ.1638 mice uniquely express an osteoblast marker and are areas of rapid/dynamic bone growth and mineralization. (a) Osteocalcin transcripts were detected in RNA recovered from NJ.1638 SNs. Total RNA from wild-type or transgenic spleen parenchyma and isolated mineralized SNs were reverse transcribed to generate template cDNA for PCR reactions using osteocalcin-specific primers (SSII+). RNA samples that were not reverse transcribed (SSII-) served as negative controls for genomic DNA contamination. Total RNA from NJ.1638 tibias and femurs served as a positive control sample for the osteocalcin primer set (294-bp RT-PCR amplicon indicated with an arrow). An actin primer set was used to demonstrate the integrity of all the samples being assayed (240-bp RT-PCR amplicon). DNA size standards (1-kb ladder) are indicated on the left. (b) NJ.1638 SNs are characterized by the presence of woven and lamellar bone. Evidence of matrix-embedded osteocytes and presence of woven bone were determined by examining toluidine blue-stained paraffin sections of SNs with polarized light. The birefringent areas of woven and lamellar bone result from polarized light interaction with collagen fibrils within the ossified nodule. Examples of regions of woven bone (Wo) and lamellar bone ( $\mathrm{Lm}$ ) are indicated. Scale bar $=0.1 \mathrm{~mm}$. IL-5 expression leads to a generalized perturbation of bone metabolism including accelerated bone growth and mineralization in the spleen. (c) A representative fluorescence photomicrograph of a SN from a transgenic animal administered calcein (green fluorescence, arrow) and tetracycline (yellow fluorescence, arrowhead) at the beginning and end of a 10-day interval, respectively (see Methods). Scale bar $=0.1 \mathrm{~mm}$. Label incorporation was assessed in bone samples from age-matched (5-8 months of age) wild-type $(n=6)$ and transgenic $(n=6)$ mice. These analyses are displayed graphically as histograms comparing the MAR (d) and the BFR/BS (e) of wild-type tibia and NJ.1638 SNs. Data are expressed as mean \pm SEM. ${ }^{A} P<0.05$.

ed for birefringence using polarized light microscopy. Assessments of undecalcified sections containing the splenic nodules using polarized light in combination with toluidine blue staining showed the presence of both woven (characterized by a random organization of collagen fibers) and lamellar (i.e., successive fine layers of organized collagen fibers) components within the nodule architecture. Cells seen embedded within the bone-like matrix are consistent in morphology with osteocytes (Figure 3b).

Evaluation of calcium content of transgenic spleens. Hydroxyapatite deposition within extracellular bone matrix is a distinguishing characteristic of bone devel- opment. Contact microradiography of sections from NJ.1638 spleens revealed the presence of mineralized tissue, and energy-dispersive $\mathrm{x}$-ray microanalysis of these mineralized splenic foci showed that the calci$\mathrm{um} /$ phosphate ratios were similar to the hydroxyapatite of normal cortical bone (data not shown).

The rates of splenic bone formation and matrix mineralization are more than an order of magnitude higher than skeletal long bone

The development of bone nodules in the spleen of transgenic mice suggests that elevated levels of IL-5 modulate bone metabolism. Histomorphometric 
parameters of bone growth and mineralization were measured in cohorts $(n=6)$ of transgenic and wild-type mice by successive administration of calcein and tetracycline to double-label the mineralizing front and allow dynamic interpretation of bone turnover. The distance between areas of label incorporation (representing the timed interval of drug administration) was measured as an estimate of appositional bone growth over time. A representative example of calcein/tetracycline incorporation in a splenic nodule is shown in Figure $3 \mathrm{c}$. Specific measures of bone growth/mineralization parameters derived from these data are shown in the histograms of Figure 3, d and e. The mineral apposition rate (MAR), the rate at which the zone of mineralization advances, in SNs $(6.36 \mu \mathrm{m} / \mathrm{d} \pm 2.61)$ was more than 30 -fold greater than the rates measured in the long bones of either wild-type $(0.22 \mu \mathrm{m} / \mathrm{d} \pm 0.04)$ or transgenic $(0.18 \mu \mathrm{m} / \mathrm{d} \pm 0.05)$ mice. Moreover, the bone formation rate corrected for bone surface (BFR/BS) in the SNs $\left(0.87 \mathrm{~mm}^{3} / \mathrm{mm}^{2} /\right.$ year \pm 0.64$)$ was also dramatically elevated relative to the rates in the tibia of wildtype $\left(0.014 \mathrm{~mm}^{3} / \mathrm{mm}^{2} /\right.$ year \pm 0.003$)$ or NJ.1638 $(0.005$ $\mathrm{mm}^{3} / \mathrm{mm}^{2} /$ year \pm 0.001$)$ mice.

\section{IL-5-mediated perturbations of bone homeostasis were not limited to the spleen}

Assessment of cancellous/trabecular bone volume (relative to cortical bone volume) in the tibias of transgenic mice $(n=10)$ revealed that the cancellous/trabecular volume of transgenic skeletal bone increased nearly twofold compared with wild-type $(n=9)$ levels (i.e., $7.86 \% \pm 1.15$ vs. $4.19 \% \pm 0.78$, respectively; $P<0.05$ ) (Figure 4a). This increase in bone volume is reflected in histological changes occurring in the femurs and tibias of transgenic mice of similar age ( $~ 8$ months of age). Representative longitudinal sections demonstrate that, unlike wild-type femur (Figure 4b), the femoral medullary cavity of NJ.1638 mice is occupied by numerous interconnecting trabeculae consistent in appearance with cancellous bone (Figure 4c). It is note- worthy that the histological changes observed in the long bones occurred concurrently with the formation of SNs, appearing 2-4 months after birth.

\section{IL-5-induced perturbations of bone-regulating factors in NJ.1638 mice are limited to acute-phase and Th2 cytokines}

IL-5 overexpression had only limited effects on a wide array of factors/signals that potentially mediate induced effects on bone metabolism, particularly heterotropic ossification of the spleen. For example, assessments of gene expression, using RT-PCR and primer sets specific for genes previously demonstrated to be associated with bone formation and/or growth, did not identify candidate genes that were upregulated in the spleen as a consequence of IL-5 overexpression (data not shown). The candidate genes examined included TGF- $\beta$ (4), leukemia inhibitory factor (LIF; ref. 36), BMP-2, BMP-4, and BMP-6 (11), Cbfa-1/Osf-2 (37), and SHh (38). Furthermore, serum levels of IL-1 $\beta$, a bone metabolism-modulating cytokine (8), remained at undetectable levels in both wild-type and transgenic mice. In contrast, IL-5 overexpression led to substantive increases in the serum levels of two cytokines, IL-4 and IL-6, both of which have been implicated as modulators of bone homeostasis $(7,8)$. Circulating IL-4 serum levels in transgenic animals $(n=4)$ were increased 10 -fold relative to age-matched wild-type littermates $(n=4), 618 \mathrm{pg} / \mathrm{ml} \pm 85$ vs. $62 \mathrm{pg} / \mathrm{ml} \pm 9$, respectively. IL-6 levels were increased from an undetectable level in wild-type mice $(n=6)$ to $76 \mathrm{pg} / \mathrm{ml} \pm 7$ in NJ.1638 animals $(n=6)$.

Splenic ossification and perturbations of skeletal long bone homeostasis occur in wild-type recipient mice engrafted with $\mathrm{NJ} .1638$ bone marrow

Potential mechanisms of IL-5-induced bone growth were assessed by adoptive engraftment of NJ.1638 bone marrow into lethally irradiated wild-type recipients. Ten days after transplantation, neither peripheral

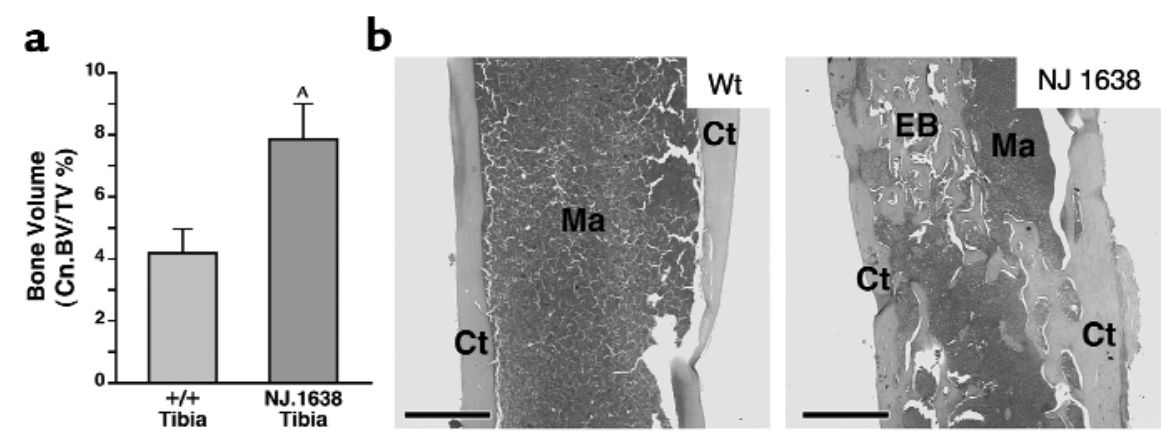

\section{Figure 4}

Perturbations of bone growth occur in the long bones of NJ.1638 mice. (a) Comparison of cancellous/trabecular bone volume versus total bone volume (Cn.BV/TV\%) of wild-type $(n=9)$ and NJ.1638 $(n=10)$ tibia. Data are expressed as mean \pm SEM. AP $<0.05$. Representative photomicrographs of hematoxylin/eosin-stained longitudinal sections of femurs from age- and sex-matched (8.5-month-old female mice) wild-type (b) and NJ.1638 (c) mice demonstrate that constitutive IL-5 expression leads to an increase in cancellous bone and occlusion of the marrow-containing lumen. Scale bars $=0.5 \mathrm{~mm}$. 

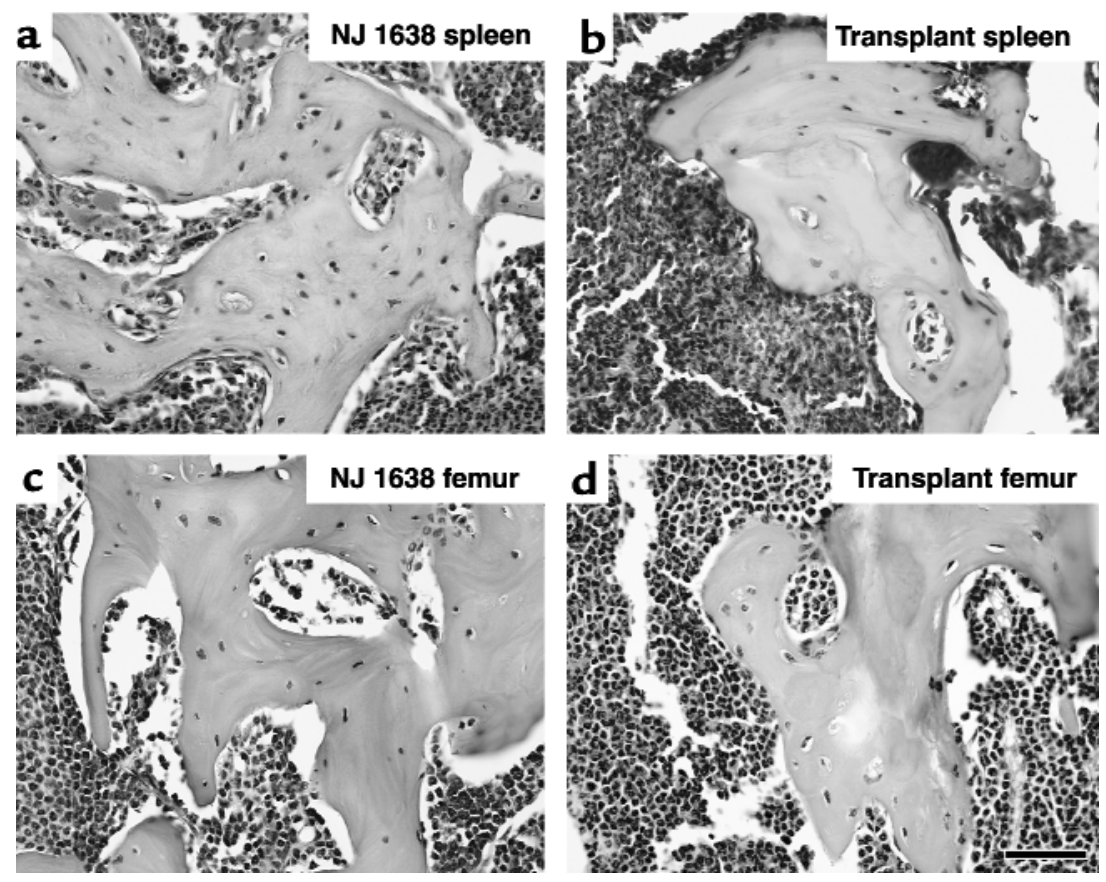

\section{Figure 5}

Splenic ossification and perturbations of skeletal long bone homeostasis occur in lethally irradiated wild-type mice after adoptive engraftment of NJ.1638 marrow. Representative photomicrographs of hematoxylin/eosin-stained decalcified sections from 8.5-month-old NJ.1638 spleen (a) and femur (c) are shown in comparison to sections of spleen (b) and femur (d) from irradiated wild-type mice 8-10 months after adoptive engraftment of NJ.1638 marrow. The striking histological similarities between the splenic and femoral ossification, regardless of the source animal, reflect both the likelihood of a single mechanism mediating bone formation as well as the marrow-derived nature of these osteogenic progenitors. Scale bars $=50 \mu \mathrm{m}$. Ct, cortical bone; Ma, bone marrow; EB, ectopic bone.

blood nor femoral marrow had more than $1 \%$ cells of the eosinophil lineage; however, eosinophils and their progenitor cells comprised greater than $60 \%$ of femoral marrow and $40 \%$ of peripheral blood 31 days after transplantation. Wild-type mice engrafted with NJ.1638 marrow cells also displayed expanded sites of hematopoiesis, including splenomegaly, and the agedependent increases in serum IL-5 levels (albeit at lower absolute levels) observed in unmanipulated NJ.1638 mice. Moreover, after engraftment, the chimeric mice developed splenic nodules in a time frame consistent with unmanipulated transgenic animals (i.e., 4-8 months). The frequency and distribution of nodules in the spleens of engrafted mice appeared indistinguishable from those of nodules of unmanipulated NJ.1638 animals, and histological characterization showed that nodules from either source were morphologically identical (Figure 5, compare a with b). The engrafted mice also displayed the perturbations of skeletal long bone homeostasis characteristic of NJ.1638 animals. In both groups of mice, increases in cancellous bone volume led to bony occlusions within the femoral cavity (Figure 5, compare $c$ with $d$ ), generating structures that were astonishingly similar to the splenic foci.

\section{Discussion}

Serum levels of IL-5 remain nearly undetectable in wildtype mice regardless of age $(<10 \mathrm{pg} / \mathrm{ml})$, whereas in NJ.1638 transgenic animals, serum IL-5 is elevated to
$800 \mathrm{pg} / \mathrm{ml}$ by 1 month of age and then slowly decreases over the next few months to a steady-state level of $\geq 400$ $\mathrm{pg} / \mathrm{ml}$. This constitutive expression of IL- 5 results in unique effects on bone metabolism, resulting in the formation of ectopic bone nodules in the spleen and increased cancellous bone growth in skeletal long bones. Because woven bone, which is found in states of rapid bone growth or high bone turnover, is formed initially followed by remodeling into lamellar bone, the presence of both lamellar and woven bone in NJ.1638 spleens suggests that this extramedullary ossification is an active and rapid event. Moreover, the histomorphometric measurements and structural organization of the induced SNs are consistent with a dynamic and regulated process. The concomitant appearance of these boneforming activities, and the expansion of extramedullary hematopoietic activity in the spleen, suggest that the perturbation of the splenic hematopoietic microenvironment is linked to ectopic ossification.

The two prominent bone-forming pathways in vivo, endochondral and intramembranous ossification, result from the differentiation of pluripotential mesenchymal cells into either chondrogenic or osteogenic lineages, respectively. The staining methodologies utilized in this study did not detect evidence of cartilaginous structures associated with the SNs, yet demonstrated the appearance of numerous foci of osteoid. This suggests that bone formation in the transgenic spleen occurs predominantly by the process of 
intramembranous ossification. The $\mathrm{BFR} / \mathrm{BS}$ and MAR of the SNs are approximately 60 -fold and 30fold higher, respectively, than the rates measured for wild-type long bones (i.e., tibia). As a consequence, bone formation and subsequent mineralization of the nodules may be occurring so rapidly that newly formed cartilage is immediately replaced by bone matrix, thus precluding the identification of cartilage in the spleens of transgenic mice.

In a direct mechanistic model, overexpression of IL-5 induces the primitive mesenchymal/osteoprogenitor target cells to undergo osteoblast differentiation, or acts directly on osteoclasts to decrease their numbers and/or impair their bone-destroying activities. Available data, however, fail to support such a direct model, as IL-5 receptors have not been reported on either osteoblasts or osteoclasts. A more plausible explanation of IL-5-mediated effects lies in an indirect model. In this model, IL-5 provides the initiating event for one or more cell types to produce signals (e.g., cytokines and growth factors) or to promote cell-cell inductive interactions that, in turn, stimulate a pathway(s) leading to osteoblast differentiation and/or decreased osteoclast activity. The two previously identified cellular targets of IL-5 in mice are B cells (39) and eosinophils (40). However, the prominent effector functions of these leukocytes, antibody production by $B$ cells or secretion of cationic secondary granule proteins by eosinophils, do not suggest an immediate relationship with bone metabolism. Nonetheless, specific mechanisms leading to enhanced bone growth arise from other activities associated with each of these cell types. For example, lymphoproliferative disorders have been associated with hypercalcemia (41), and B cells have been shown to secrete $\mathrm{Th} 2$ inflammatory cytokines that also have direct effects on bone through the alteration of osteoclast function (e.g., IL-4 [refs. 14, 42] and IL-10 [refs. 43, 44]). Moreover, the lack of histological evidence (e.g., acid phosphatase staining) showing significant numbers of osteoclasts specifically associated with the nodules (data not shown) is consistent with a mechanism of bone formation dependent on IL-5/B cell-mediated suppression of osteoclasts and/or osteoclast activity. Alternatively, both B cells and eosinophils have each been shown to express a wide range of other cytokines $(45,46)$ and metabolic intermediates/growth factors (47) that may contribute to a "multifactoral milieu" directly stimulating bone formation. The potential role of eosinophils is of particular interest because of the dramatic expansion of this cell type in the spleens of transgenic animals and the ability of these cells to secrete signals that have direct effects on osteoblasts (e.g., TGF- $\beta$ [refs. 4, 48] and LIF [refs. 36, 49]). It is noteworthy that although a RT-PCR assay was unable to detect elevated levels of TGF- $\beta$ transcripts in transgenic spleens, elevated levels of activated TGF- $\beta$ (relative to wild-type) were detected in NJ.1638 spleen explants (data not shown). This observation suggests that eosinophils have the potential to elicit bone formation through the release and processing of stored inactive TGF- $\beta$.
The effects of IL-5 overexpression in NJ.1638 animals were not limited to the de novo appearance of bone in the spleen, but also included systemic changes in bone metabolism that affected the skeletal long bones. These changes resulted in a significant increase in the cancellous bone volume (Cn.BV/TV\%) of tibias and the occlusion of the marrow cavity in femurs of transgenic mice. Interestingly, the increase in skeletal bone volume was not accompanied by equivalent changes in the MAR or BFR/BS, implying that IL-5 activities are limited to effects on osteoblast numbers (i.e., recruitment and/or differentiation). Existence of a much higher rate of bone formation in transgenic spleen, in comparison to either wild-type and transgenic long bones, is likely a consequence of the chaotic nature of splenic bone formation. The nodules in the spleen are small irregularly shaped foci (i.e., very high volume/surface area ratios) and are numerous. Furthermore, the Goldner'sstained sections of the nodules showed that each of these foci display one or more areas of active bone formation (i.e., associated area[s] of osteoid). Thus, relative to these numerous and spontaneously forming nodules in the spleen, active areas of osteogenesis (i.e., the net rate of bone formation/total bone surface) in highly ordered structures such as the tibia will be restricted regardless of the actions of IL-5.

The ability of NJ.1638 marrow to mediate ectopic osteogenesis upon adoptive engraftment into lethally irradiated wild-type mice suggests that IL-5-dependent differentiation of resident blast cells was not occurring, as the hematopoietic and trabecular compartments of the recipient spleens were destroyed or, at a minimum, were rendered nonproliferative by irradiation. These data imply that bone formation in transgenic mice occurs as a consequence of IL-5-mediated mobilization of marrow-derived osteoprogenitor cells and/or IL5 -mediated suppression of recruited osteoclasts. Furthermore, these mechanisms are not mutually exclusive, as IL-5 may mediate both the splenic recruitment and activation of osteoblasts, as well as the attenuation of the bone-destroying activities of recruited splenic osteoclasts. The implied proliferation and recruitment of marrow-derived osteoblast/osteoclast progenitor cells is supported by our earlier studies of NJ.1638 mice, which demonstrated that elevated IL-5 levels led to the mobilization of both erythropoietic and megakaryocytic stem cells from the marrow to the spleen (25). In addition, other engraftment studies have shown that IL-5 overexpression leads to the mobilization of early hematopoietic stem cells from the marrow to the liver (our unpublished observations). Collective$\mathrm{ly}$, these observations suggest that, in addition to activities on eosinophilopoiesis, a primary effector function of IL-5 is to mobilize marrow-derived stem cells in support of extramedullary sites of hematopoiesis. The results presented here show that this IL-5-mediated mobilization may also extend to marrow-derived osteogenic stromal cells and osteoclast progenitors, possibly reflecting coordinate signaling between these 
cell populations and the increased extramedullary hematopoietic activities occurring in transgenic mice. The striking histological similarities shared by the ossified foci of the spleen and the bony network within the femoral lumen suggest that the different manifestations of IL-5-mediated bone formation result from a single mechanism. The demonstration of IL-5-mediated increases in serum levels of cytokines with osteogenic activities implies that this mechanism of ossification relies on the release of a soluble factor(s). The implicit conclusion of such a mechanism is that the cell/tissue specificity of IL-5 expression is not relevant, and increased levels of IL-5 alone will lead to the elaboration of one or more soluble factors affecting bone homeostasis. Observations of equivalent splenic ossification occurring in several other transgenic mouse lines overexpressing IL-5 from non-T cell sources at even higher serum levels than NJ.1638 (e.g., alveolar type II and keratinocyte specific [our unpublished observations]) support this conclusion. Thus, the in vivo osteogenic importance of elevated IL-5 levels appears to be independent of both the cell source and absolute level of this cytokine.

The linkage of IL-5 expression and bone homeostasis implicates this cytokine as a heretofore unknown regulator of bone metabolism. Although the effects of IL-5 overexpression are pleiotropic, eliciting both the expected expansion of eosinophil and B cell populations as well as augmenting bone deposition, these effects may not be independent events, and IL-5-induced changes in bone metabolism may occur as a consequence of eosinophil- and/or B cell-mediated activity(ies).

\section{Acknowledgments}

We are grateful to Thomas Spelsberg, Russell Turner, and Theresa Hefferan for their critical comments and advice. We thank Gloria Yueh and David Gardner for assistance with the whole-mount staining protocol, and Merry Jo Oursler for measurements of activated TGF- $\beta$. Invaluable assistance was provided by the Mayo Clinic Scottsdale Histology Core Facility (Director: Anita Jennings), Mayo Clinic Rochester Bone Histomorphometry Laboratory, and the Mayo Clinic Scottsdale Graphic Arts Facility (Director: Marv Ruona). We also extend a heartfelt thanks to our research program assistant, Linda Mardel, whose patience and efforts helped turn our science into a readable manuscript. This work was supported by the Mayo Foundation and a National Research Service Award (M. Macias) from the National Heart, Lung, and Blood Institute (F32HL10105).

1. Garcia-Ojeda, M.E., Dejbakhsh-Jones, S., Weissman, I.L., and Strober, S 1998. An alternate pathway for T cell development supported by the bone marrow microenvironment: recapitulation of thymic maturation. J. Exp. Med. 187:1813-1823.

2. Taichman, R.S., Reilly, M.J., and Emerson, S.G. 1996. Human osteoblasts support human hematopoietic progenitor cells in vitro bone marrow cultures. Blood. 87:518-524.

3. Aubin, J.E. 1999. Osteoprogenitor cell frequency in rat bone marrow stromal populations: role for heterotypic cell-cell interactions in osteoblast differentiation. J. Cell. Biochem. 72:396-410.

4. Andrades, J.A., et al. 1999. A recombinant human TGF-beta1 fusion pro- tein with collagen-binding domain promotes migration, growth, and differentiation of bone marrow mesenchymal cells. Exp. Cell Res. 250:485-498.

5. Prockop, D.J. 1997. Marrow stromal cells as stem cells for nonhematopoietic tissues. Science. 276:71-74.

6. Pereira, R.F., et al. 1995. Cultured adherent cells from marrow can serve as long-lasting precursor cells for bone, cartilage, and lung in irradiated mice. Proc. Natl. Acad. Sci. USA. 92:4857-4861.

7. Manolagas, S.C., and Jilka, R.L. 1995. Bone marrow, cytokines, and bone remodeling. Emerging insights into the pathophysiology of osteoporosis. N. Engl. J. Med. 332:305-311.

8. Lorenzo, J.A. 1991. The role of cytokines in the regulation of local bone resorption. Crit. Rev. Immunol. 11:195-213.

9. Van Vlasselaer, P., Falla, N., Van Den Heuvel, R., Dasch, J., and de Waal Malefijt, R. 1995. Interleukin-10 stimulates hematopoiesis in murine osteogenic stroma. Clin. Orthop. 313:103-114.

10. Christenson, R.H. 1997. Biochemical markers of bone metabolism: an overview. Clin. Biochem. 30:573-593.

11. Wozney, J.M. 1998. The bone morphogenetic protein family: multifunctional cellular regulators in the embryo and adult. Eur. J. Oral Sci. 106(Suppl. 1):160-166.

12. Kingsley, D.M. 1994. What do BMPs do in mammals? Clues from the mouse short-ear mutation. Trends Genet. 10:16-21.

13. Van Vlasselaer, P., Borremans, B., van Gorp, U., Dasch, J.R., and De WaalMalefyt, R. 1994. Interleukin 10 inhibits transforming growth factorbeta (TGF-beta) synthesis required for osteogenic commitment of mouse bone marrow cells. J. Cell Biol. 124:569-577.

14. Lewis, D.B., et al. 1993. Osteoporosis induced in mice by overproduction of interleukin 4. Proc. Natl. Acad. Sci. USA. 90:11618-11622.

15. Leng, S.X., and Elias, J.A. 1997. Interleukin-11. Int. J. Biochem. Cell Biol. 29:1059-1062.

16. Ershler, W.B., Harman, S.M., and Keller, E.T. 1997. Immunologic aspects of osteoporosis. Dev. Comp. Immunol. 21:487-499.

17. Caplan, A.I., and Boyan, B.D. 1994. Endochondral bone formation: the lineage cascade. In Mechanisms of bone development and growth. Volume 8. B.K. Hall, editor. CRC Press. Boca Raton, Florida, USA. 1-46.

18. Kaddu, S., et al. 1995. Extramedullary hematopoiesis in pilomatricomas. Am. J. Dermatopathol. 17:126-130.

19. Sleater, J., Mullins, D., Chun, K., and Hendricks, J. 1996. Fibro-osseous pseudotumor of the digit: a comparison to myositis ossificans by light microscopy and immunohistochemical methods. J. Cutan. Pathol. 23:373-377.

20. Rooney, P. 1994. Intratendinous ossification. In Mechanisms of bone development and growth. Volume 8. B.K. Hall, editor. CRC Press. Boca Raton, Florida, USA. 47-83.

21. Woolley, K., and Stark, P. 1999. Pulmonary parenchymal manifestations of mitral valve disease. Radiographics. 19:965-972.

22. Nabili, V., Brodie, H.A., Neverov, N.I., and Tinling, S.P. 1999. Chronology of labyrinthitis ossificans induced by Streptococcus pneumoniae meningitis. Laryngoscope. 109:931-935.

23. Baysal, T., Elmali, N., Kutlu, R., and Baysal, O. 1998. The stone man: myositis (fibrodysplasia) ossificans progressiva. Eur. Radiol. 8:479-481.

24. Shafritz, A.B., et al. 1996. Overexpression of an osteogenic morphogen in fibrodysplasia ossificans progressiva. N. Engl. J. Med. 335:555-561.

25. Lee, N.A., et al. 1997. Expression of IL-5 in thymocytes/T cells leads to the development of a massive eosinophilia, extramedullary eosinophilopoiesis, and unique histopathologies. J. Immunol. 158:1332-1344.

26. Lee, N.A., Loh, D.Y., and Lacy, E. 1992. CD8 surface levels alter the fate of $\alpha \beta$ TCR expressing thymocytes in transgenic mice. J. Exp. Med. 175:1013-1025.

27. Kaufman, M.H. 1992. The atlas of mouse development. Academic Press. New York, New York, USA. 291 pp.

28. Ingram, R.T., Collazo-Clavell, M., Tiegs, R., and Fitzpatrick, L.A. 1996. Paget's disease is associated with changes in the immunohistochemical distribution of noncollagenous matrix proteins in bone. J. Clin. Endocrinol. Metab. 81:1810-1820.

29. Clarke, B.L., et al. 1996. Changes in quantitative bone histomorphometry in aging healthy men. J. Clin. Endocrinol. Metab. 81:2264-2270.

30. Fitzpatrick, L.A., Severson, A., Edwards, W.D., and Ingram, R.T. 1994. Diffuse calcification in human coronary arteries. Association of osteopontin with atherosclerosis. J. Clin. Invest. 94:1597-1604.

31. Parfitt, A.M., et al. 1987. Bone histomorphometry: standardization of nomenclature, symbols, and units. Report of the ASBMR Histomorphometry Nomenclature Committee. J. Bone Miner. Res. 2:595-610.

32. Baylink, D., Stauffer, M., Wergedal, J., and Rich, C. 1970. Formation, mineralization, and resorption of bone in vitamin D-deficient rats. J. Clin. Invest. 49:1122-1134.

33. Fitzpatrick, L.A., and Khurana, J.S. 2000. Bone histomorphometry and undecalcified bone biopsies. In A compendium of skeletal pathology. J.S. Khurana et al., editors. United Pathologists Press. 53-58. Seoul, Korea.

34. Novak, E.K., et al. 1995. Inherited thrombocytopenia caused by reduced platelet production in mice with the gunmetal pigment gene mutation. 
Blood. 85:1781-1789.

35. Malaval, L., Liu, F., Roche, P., and Aubin, J.E. 1999. Kinetics of osteoprogenitor proliferation and osteoblast differentiation in vitro. J. Cell. Biochem. 74:616-627.

36. Auernhammer, C.J., and Melmed, S. 2000. Leukemia-inhibitory factorneuroimmune modulator of endocrine function. Endocr. Rev. 21:313-345.

37. Ducy, P., Zhang, R., Geoffroy, V., Ridall, A.L., and Karsenty, G. 1997. Osf2/Cbfa1: a transcriptional activator of osteoblast differentiation. Cell. 89:747-754.

38. Iwamoto, M., Enomoto-Iwamoto, M., and Kurisu, K. 1999. Actions of hedgehog proteins on skeletal cells. Crit. Rev. Oral Biol. Med. 10:477-486.

39. Harada, N., Kikuchi, Y., Tominaga, A., Takaki, S., and Takatsu, K. 1985. BCGFII activity on activated B cells of a purified murine T cell-replacing factor (TRF) from a $\mathrm{T}$ cell hybridoma (B151K12). J. Immunol. 134:3944-3951.

40. Sanderson, C.J., Warren, D.J., and Strath, M. 1985. Identification of lymphokine that stimulates eosinophil differentiation in vitro. Its relationship to interleukin 3, and functional properties of eosinophils produced in cultures. J. Exp. Med. 162:60-74.

41. Daroszewska, A., Bucknall, R.C., Chu, P., and Fraser, W.D. 1999. Severe hypercalcaemia in B-cell lymphoma: combined effects of PTH-rP, IL-6 and TNF. Postgrad. Med. J. 75:672-674.

42. Lacey, D.L., et al. 1995. Interleukin 4, interferon-gamma, and prostaglandin $\mathrm{E}$ impact the osteoclastic cell-forming potential of murine bone marrow macrophages. Endocrinology. 136:2367-2376.

43. Owens, J.M., Gallagher, A.C., and Chambers, T.J. 1996. IL-10 modulates formation of osteoclasts in murine hemopoietic cultures. J. Immunol. 157:936-940

44. Hong, M.H., Williams, H., Jin, C.H., and Pike, J.W. 2000. The inhibitory effect of interleukin-10 on mouse osteoclast formation involves novel tyrosine-phosphorylated proteins. J. Bone Miner. Res. 15:911-918.

45. Rumbley, C.A., et al. 1999. Activated eosinophils are the major source of Th2-associated cytokines in the schistosome granuloma. J. Immunol. 162:1003-1009.

46. Charyulu, V.I., and Lopez, D.M. 2000. Elevated GM-CSF levels in tumor bearing mice upregulate IL- 6 production by B cells via a mechanism independent of TNF-alpha. Int. J. Oncol. 16:161-167.

47. Weller, P.F. 1994. Eosinophils: structure and functions. Curr. Opin. Immunol. 6:85-90.

48. Eisma, R.J., Allen, J.S., Lafreniere, D., Leonard, G., and Kreutzer, D.L. 1997. Eosinophil expression of transforming growth factor-beta and its receptors in nasal polyposis: role of the cytokines in this disease process. Am. J. Otolaryngol. 18:405-411.

49. Zheng, X., et al. 1999. Leukemia inhibitory factor is synthesized and released by human eosinophils and modulates activation state and chemotaxis. J. Allergy Clin. Immunol. 104:136-144. 